\title{
Regioselective cationization of cellulosic materials using an efficient solvent-minimizing spray-technique
}

\author{
Hanna de la Motte • Gunnar Westman
}

Received: 22 February 2012/Accepted: 23 July 2012/Published online: 9 August 2012

(C) The Author(s) 2012. This article is published with open access at Springerlink.com

\begin{abstract}
Cationization of different cellulosic materials was successfully accomplished using an efficient solvent-minimizing spray-technique. The obtained materials were studied and evaluated in regard to reaction efficiency and regioselectivity using $1 \mathrm{D}$ and ${ }^{1} \mathrm{H}-{ }^{13} \mathrm{C}$ correlated 2D NMR experiments. The high consistencies and temperatures applied using the spraytechnique resulted in reagent effective etherifications and shorter reaction times. The NMR spectra indicated that the spray-technique favors substitutions at position O-6, showing a regioselectivity of O-6 $>$ O-2 $>$ O-3 for softwood kraft pulp (SKP) and cotton linters. However, cationization of mercerized cellulose and dissolving pulp, using the spray-technique, demonstrated more efficient reactions compared to SKP and cotton, and a regioselectivity of O-6 $\geq 0-2>0-3$. Nanocrystalline cellulose showed the lowest reactivity and a regioselectivity of O-6 $\gg$ O-2 $>$ O-3. In this work we provide information on structure-property relationships and characterization methods for modified polysaccharides, together with a solvent system that is well known industrially and in line with sustainability aspects.
\end{abstract}

Electronic supplementary material The online version of this article (doi:10.1007/s10570-012-9755-8) contains supplementary material, which is available to authorized users.

H. de la Motte $(\bowtie) \cdot$ G. Westman

Organic Chemistry, Department of Chemical and

Biological Engineering, Chalmers University of

Technology, 41296 Göteborg, Sweden

e-mail: hanna.delamotte@chalmers.se
Keywords Cationization - Softwood kraft pulp · Nanocrystalline cellulose · Dissolving pulp · Regioselectivity $\cdot$ NMR characterization

\section{Introduction}

The inherent polyhydroxylic structure of polysaccharide polymers enables functionalization by a wide selection of chemical reactions. The physical and chemical properties obtained after modifications are highly dependent on how and where the substituents are located along the polymer chains, an issue which has been widely investigated by several research groups (Klemm 1998; Mischnick and Momcilovic 2010). These findings, and the increased attention on new sustainable materials, have thus resulted in a growing interest in controlled modifications and characterization methods appropriate for polysaccharides.

Cationization is an important category of commercial polysaccharide derivatizations involving functional products such as pollutant adsorbers, paper additives, antibacterial agents in food and medicine and conditioners for cosmetic applications (Belalia et al. 2008; Peniche and Peniche 2011; Saito and Isogai 2007; Yan et al. 2009). Preferred industrial quarternization methods for cellulose are to attach a pre-built quaternary ammonium moiety to the cellulosic polymer using either of the two most common synthetic routes for industrial etherifications of cellulose: the Williamson ether synthesis, reacting cellulose alkoxides with alkyl 
halides, and the ring-opening reaction of alkylene oxides, epoxides, with alkali activated hydroxy groups. These reactions are performed in heterogeneous systems using aqueous alkaline solutions, conditions that often results in inefficient reactions with low reagent yields and less control of the substitutions (Antal and Micko 1992; Klemm et al. 1998; Lim and Hudson 2004). Recently more efficient and controlled reactions in organic/base systems have been reported resulting in well defined cellulosic ethers with specific substitution patterns (Baumann et al. 2003; Fox et al. 2011; Heinze and Liebert 2001; Petzold et al. 2003; Volkert and Wagenknecht 2008; Heinze and Petzold 2008). However, in addition to new unconventional methods, aqueous alkaline solutions as solvent systems are well known industrially and in line with sustainability aspects (Hashem et al. 2003).

In this work, cationizations using the epoxide 2,3epoxypropyltrimethylammonium chloride (EPTMAC), have been investigated in detail using a solvent-minimizing spray-technique. The method has been evaluated regarding efficiency and distributions of the substituents in order to expand the knowledge of structure-property relationships in the synthesis of cellulose ethers. Previous findings have indicated that the spray-technique is an efficient etherification method compared to other heterogeneous aqueous systems, and results in different substitutions pattern (de la Motte et al. 2011). This paper verifies the efficiency and regioselectivity of the spraytechnique on two cellulosic materials, softwood kraft pulp (SKP) and cotton, and investigates the influence of consistency and temperature further.

Additionally, the impact of the spray-technique on cellulosic materials with different macromolecular structures, such as nanocrystalline cellulose, mercerized cellulose and dissolving pulp was examined. Decrystallization is a utilized method to increase cellulose reactivity (Buschlediller and Zeronian 1992) and for this reason, cellulosic materials with different pre-treatments and crystallinities were investigated using the spray-technique.

\section{Materials and methods}

\section{Materials}

A never-dried, industrially produced Scandinavian SKP bleached in a totally chlorine free peroxide-based sequence was used in the experiments. Nanocrystalline cellulose was prepared by sulphuric acid hydrolysis of the SKP according to the previously reported procedure (Beck-Candanedo et al. 2005). The cotton linters were obtained from Munktell Filter $\mathrm{AB}$, Sweden. 2,3-epoxypropyltrimethylammonium chloride (EPTMAC) was obtained from Shandong Guofeng Fine Chemical Co., Ltd, China, and used without further purification. The cationic exchange sorbent ISOLUTE $^{\circledR}$ CBA was obtained from Sorbent AB, Sweden. The kraft dissolving pulp was delivered from Bahia pulp SA, with a degree of polymerization of 1,340 .

Methods

Etherification of cellulosic materials using a solventminimizing spray-technique, method MI

The modification was performed according to previous reported protocol (de la Motte et al. 2011): The fibers $(1 \mathrm{~g})$ were activated by washing the pulp with a sodium hydroxide solution $(0.1-1 \mathrm{M})$ followed by solvent exchange with acetone. The fibers were then oven-dried at $105^{\circ} \mathrm{C}$ for $1 \mathrm{~h}$. After drying, the fibers were sprayed with EPTMAC (molar ratio EPTMAC/ anhydroglucopyranose unit $(\mathrm{AGU})=0.53-4.2)$ dissolved in water $(0.66-10 \mathrm{~mol} / \mathrm{L}$, consistencies of final mixtures $=4.8-44 \%$ ) and kneaded to distribute the reagent. The EPTMAC impregnated pulp was placed in an open bowl in an oven at $50-140{ }^{\circ} \mathrm{C}$ for $1 \mathrm{~h}$. Following heat treatment, the sample was stored over night at room temperature. The sample was then thoroughly washed with water and subjected to solvent exchange with acetone followed by air drying. The nanocrystalline cellulose was, after the storing over night, subjected to dialysis for 3 days using Spectra/ Por Dialysis Membrane (MWCO 12-14,000), filtrated and dried.

\section{Etherification of cellulosic materials in a heterogeneous alkaline aqueous system, method M2}

The modification was performed according to previous reported protocol (Hasani et al. 2009): The cellulosic materials $(1 \mathrm{~g})$ were mixed with a sodium hydroxide solution $(0.1-1 \mathrm{M})$ at a consistency of $4.8 \%$. After $30 \mathrm{~min}$ at room temperature, EPTMAC 
(molar ratio EPTMAC/AGU $=2.1$ ) was added and the temperature was increased to $50{ }^{\circ} \mathrm{C}$. After $5 \mathrm{~h}$, the reaction mixture was diluted with water. The product was filtered, washed thoroughly with distilled water and acetone, and then air-dried.

\section{Mercerization of SKP}

Softwood kraft pulp were mercerized in $30 \% \mathrm{NaOH}$ solution (cellulose:liquor ratio $1: 50 \mathrm{w} / \mathrm{w}$ ) at $0{ }^{\circ} \mathrm{C}$ for $1 \mathrm{~h}$. The mixture was then washed with water until a constant $\mathrm{pH}$ was obtained, and air-dried at room temperature. The mercerized samples were reacted according to procedure M1 after treatment.

\section{Hydrolysis}

The cationic modified cellulose substrates were hydrolyzed according to Theander and Westerlund (1986): $3 \mathrm{~mL}$ of sulfuric acid (12 M) was added to $200 \mathrm{mg}$ of cellulosic substrate. The sample was subjected to vacuum for $15 \mathrm{~min}$ and then heated in a water bath $\left(30{ }^{\circ} \mathrm{C}\right)$ for $1 \mathrm{~h}$. Distilled water $(84 \mathrm{~g})$ was added and the sample was autoclaved for $1 \mathrm{~h}$ at $125^{\circ} \mathrm{C}$. The hydrolysate was filtered, neutralized with barium hydroxide and again filtered. The filtrate was concentrated to dryness under reduced pressure.

\section{Preparative ion exchange chromatography}

The ion exchange chromatography was performed according to previous reported protocol (de la Motte et al. 2011).

\section{NMR analysis, elementary analysis and $X$-ray diffraction}

Prior to NMR analysis, the hydrolyzed materials were lyophilized and dissolved in $\mathrm{D}_{2} \mathrm{O}$. The NMR spectra were recorded at $25^{\circ} \mathrm{C}$ on a Varian 400-MR $400 \mathrm{MHz}$ spectrometer operating at $399.95 \mathrm{MHz}$ for proton detection and at $100.58 \mathrm{MHz}$ for carbon detection. The nitrogen analysis was provided by the Mikroanalytische Laboratorium, Kolbe, Germany. The X-ray diffraction results were provided from Environmental Inorganic Chemistry, Department of Chemical and Biological Engineering, Chalmers University of Technology.

\section{DS calculations}

The degree of substitution was estimated from NMR spectra according to the following equation:

$$
\begin{aligned}
& D S_{N M R} \\
& =\frac{\int(\beta H 2+9 \text { methyl protons of EPTMAC })-\int \beta H 1}{9 \times\left(\int \alpha H 1+\int \beta H 1\right)}
\end{aligned}
$$

where $\int \beta H 2=\int \beta H 1=1$ is the normalized integrated area of the $\beta-\mathrm{H} 1$ peak.

The degree of substitution was calculated from the nitrogen analysis according to the following equation:

$$
D S_{N}=\frac{162.15 \times \% N}{1401-151.64 \times \% N}
$$

\section{Result and discussion}

Previous findings have indicated that the solventminimizing spray-technique is an efficient etherification method and could result in different substitutions pattern (de la Motte et al. 2011). To further investigate these findings, etherifications using the spray-technique (M1) and a more common etherification method in a heterogeneous alkaline aqueous system (M2), were performed on different cellulosic materials under various conditions. To achieve information regarding reaction efficiencies and introduction of substituents on the anhydroglucopyranose units, various high resolution 1D and 2D NMR-techniques were applied together with elementary analysis and X-ray diffraction.

Analysis using NMR spectroscopy enables evaluation of reaction efficiency and regioselectivity of the material. The efficiency of the reaction, measured as the degree of substitution ( $\mathrm{DS}_{\mathrm{NMR}}$ ), can be estimated from the $1 \mathrm{D}{ }^{1} \mathrm{H}-\mathrm{NMR}$ spectrum according to Eq. 1. To improve the accurateness of the proton integrals, a quantitative ${ }^{1} \mathrm{H}-\mathrm{NMR}$ spectrum (Fig. 1) with automatic pulse-width calibration and increased length of the first delay $(\mathrm{d} 1=50 \mathrm{~s})$ was recorded as a reference. The length of the first delay guarantees full relaxation of the molecules before the next pulse, and provides peaks with more accurate integrals. It must, however, be emphasized that $\mathrm{DS}_{\mathrm{NMR}}$ values obtained by ${ }^{1} \mathrm{H}$ NMR are non-absolute, but comparative reliable, as difficult integration of overlapping, weak or close peaks must be taken into consideration. The two peaks originating from the $\mathrm{H}_{\mathrm{a}}$-1-protons (i.e. $\mathrm{H}$-1-protons 


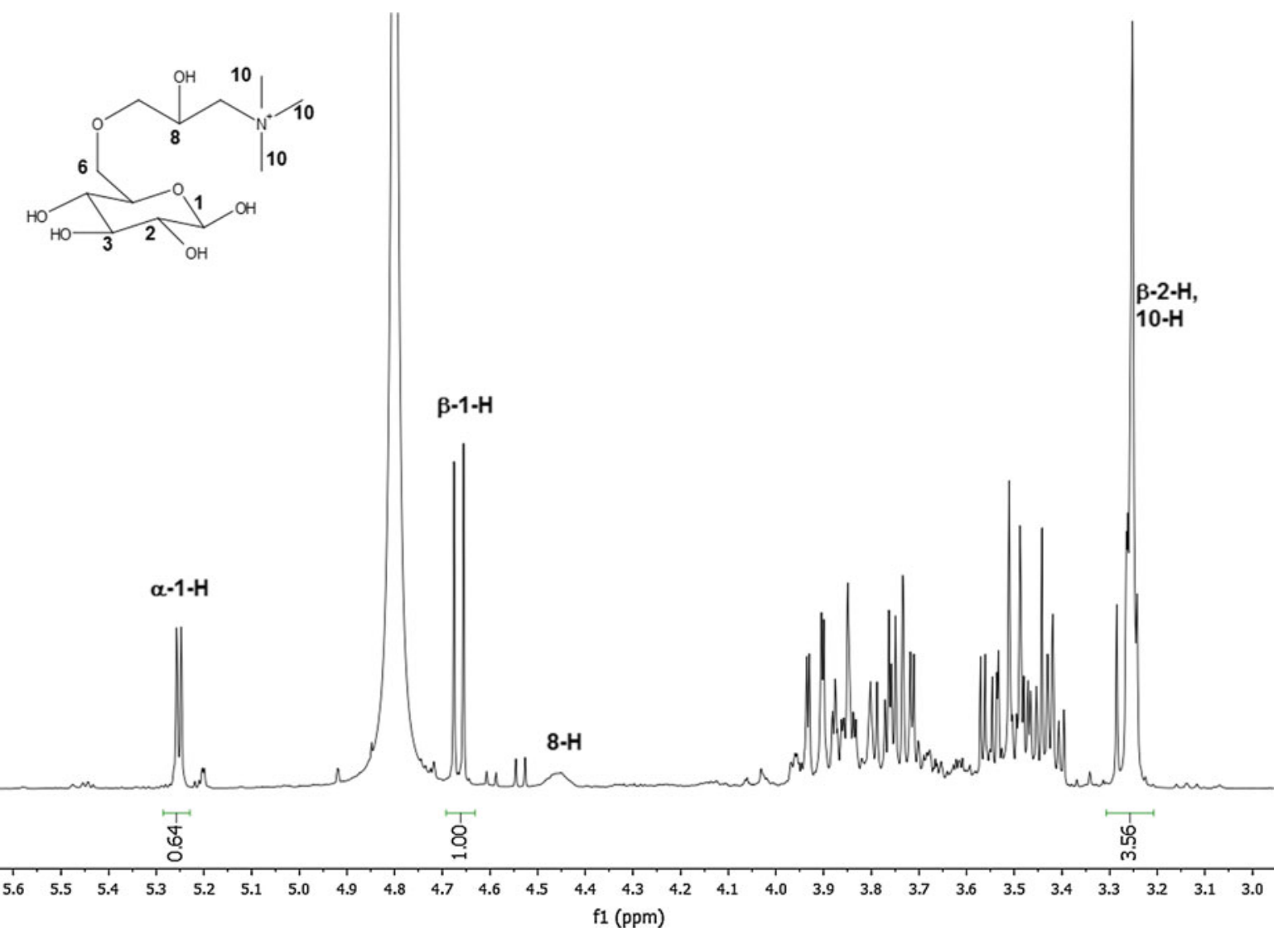

Fig. 1 A quantitative ${ }^{1} \mathrm{H}-\mathrm{NMR}$ spectrum of hydrolyzed SKP modified with the cationization reagent EPTMAC

affected by substitution at position O-2) are difficult to integrate with certainty in the ${ }^{1} \mathrm{H}-\mathrm{NMR}$ spectra. The $\beta$ $\mathrm{H}_{\mathrm{a}}-1$ (4.74 ppm) is hidden (or overlapped) in the water peak and the $\alpha \mathrm{H}_{\mathrm{a}}-1$ peak (5.45 ppm) is close to peaks belonging to hemicelluloses. These peaks are for that reason excluded in the $\mathrm{DS}_{\mathrm{NMR}}$ equation (Eq. 1), which in turn results in a slight overestimation of the $\mathrm{DS}_{\mathrm{NMR}^{-}}$ values. This overestimation of the $\mathrm{DS}_{\mathrm{NMR}^{-}}$-calculation was controlled and confirmed using elementary analysis $\left(\mathrm{DS}_{\mathrm{N}}\right)$ on two cationized samples, one of SKP and one of mercerized cellulose. For the SKP, the $\mathrm{DS}_{\mathrm{NMR}}$ value was 0.19 and the $\mathrm{DS}_{\mathrm{N}}$ value 0.16 (1.20 \pm $0.01 \% \mathrm{~N})$ and for the mercerized cellulose, the $\mathrm{DS}_{\mathrm{NMR}}$ value was 0.25 and the $\mathrm{DS}_{\mathrm{N}}$ value 0.22 (1.605 \pm $0.005 \% \mathrm{~N}$ ). However, the difference in DS between the two modified samples is similar using either of the two characterization methods, which is of importance in this paper (for details regarding the two samples used in the DS-comparison, see supporting information, Table S8).
The relative amount of substitution at positions O-2 and $\mathrm{O}-3$ can be distinguished in the ${ }^{13} \mathrm{C}-{ }^{1} \mathrm{H}$ correlated heteronuclear single quantum coherence (HSQC) NMR spectra (Fig. 2). The HSQC-NMR spectra show the typical $\sim 9$ ppm change in shift for carbons directly attached to substituted hydroxy groups, and the selectivity of the substitutions can thus be determined by comparing the integrals of the peaks belonging to un-substituted and substituted atoms. Substitution at position O-3 is normally less favored in heterogeneous reactions, which means that most of the substitutions occurs at positions O-2 or O-6.

Etherification methods and regioselectivity

In the first study, cotton linters and SKP were reacted with the cationic epoxide 2,3-epoxypropyltrimethylammonium chloride (EPTMAC) according to the two previous reported methods M1 and M2 (de la Motte et al. 2011; Hasani et al. 2009). The spray-technique 


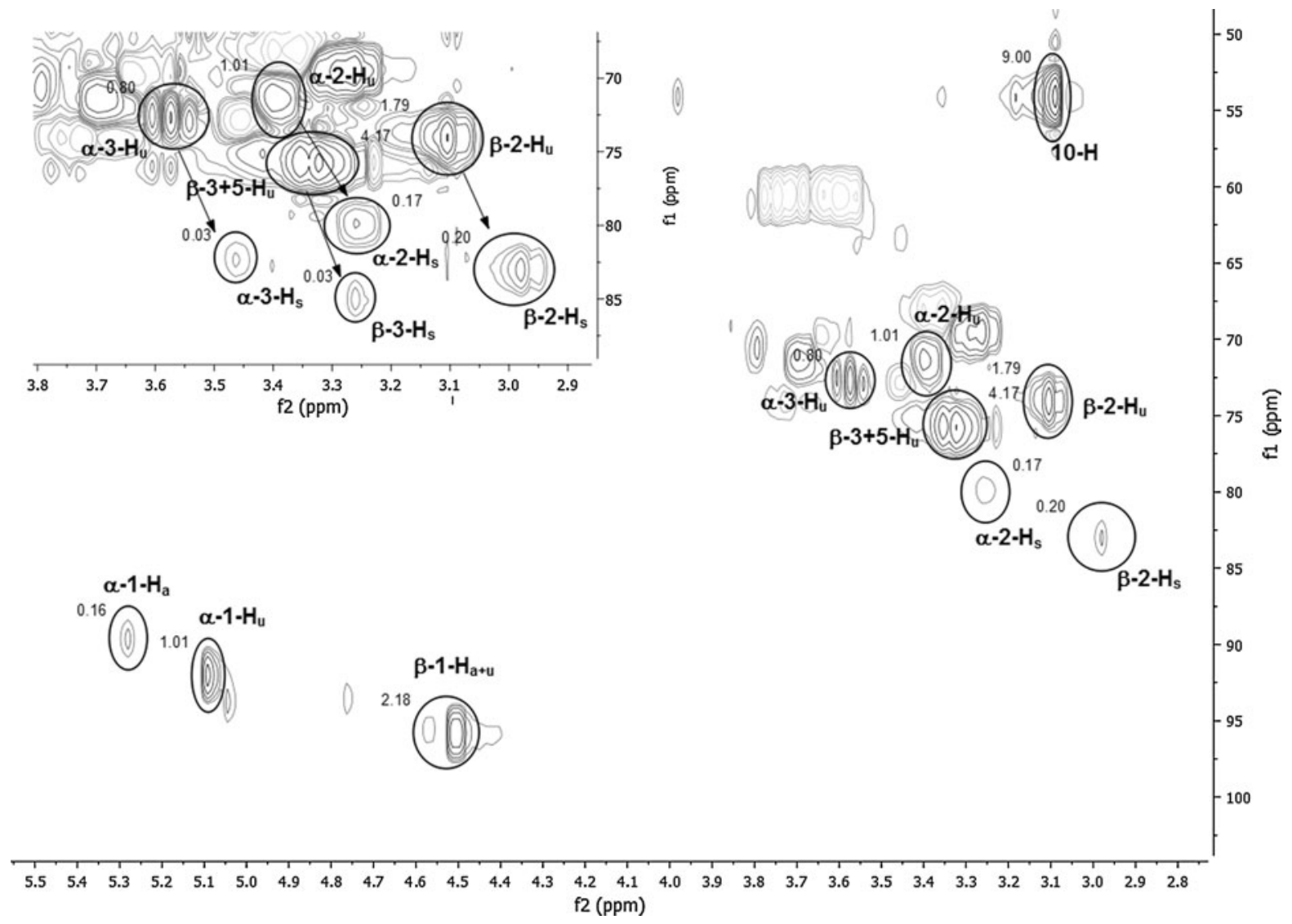

Fig. 2 HSQC-NMR spectrum of hydrolyzed mercerized cellulose modified with the cationization reagent EPTMAC. The protons located on the directly attached carbons to the unsubstituted O-2 and O-3 (- $\left.\mathrm{H}_{\mathrm{u}}\right)$, the EPTMAC protons $(10-\mathrm{H})$, the $\alpha$ and $\beta \mathrm{H}-1$ protons and the $\mathrm{H}-1$ protons affected by substitution

(M1) uses a lower amount of reagent, less solvent, higher temperature and a shorter reaction time, whereas the more common method (M2) uses a higher amount of reagent in an alkaline aqueous slurry, lower temperature and a longer reaction time.

Furthermore, the spray-method includes a preactivation step with sodium hydroxide to avoid an alkaline aqueous environment in the spraying-mixture, and executes the reaction in an open bowl inside an oven to evaporate the solvent during the reaction. These features are believed to reduce the possible hydrolysis of the epoxide reagent to the diol.

To assure full solubility in deuterated solvents, the modified cellulosic materials were subjected to complete hydrolysis using sulfuric acid prior to NMR analysis. The cationic monomers were, exclusively in this first experiments (samples 1-8), further concentrated by the use of at $\mathrm{O}-2\left(\mathrm{H}_{\mathrm{a}}-1\right)$ are integrated in the spectrum using circles. The protons located on the directly attached carbons to substituted O-2 and $\mathrm{O}-3\left(-\mathrm{H}_{\mathrm{s}}\right)$ peaks are magnified in the inserted spectrum, and the shift changes after substitution marked with arrows

preparative ion exchange chromatography to enhance the signals arising from the modified monomers.

In Table 1, the results from the first study are presented demonstrating that the solvent-minimizing spray-technique, M1, is a reagent efficient etherification method. To obtain products with the same degree of substitution $\left(\mathrm{DS}_{\mathrm{NMR}}\right)$, only a fourth of the amount of EPTMAC is required during a five times shorter reaction time.

It is also illustrated in Table 1, as have been reported previously by Heinze and Pfeiffer (1999), that an increased concentration of sodium hydroxide turns selectivity slightly towards position O-6. The same tendency regarding the selectivity can be seen when using the spray-technique M1; the substitutions at position O-2 decreases consistently using the spraytechnique. 
Table 1 Influence of reaction conditions and methods, using the spray-technique (M1) or a heterogeneous alkaline aqueous system (M2), on the etherification of cotton linters (CL) and softwood kraft pulp (SKP) with the cationic molecule 2,3epoxypropyltrimethylammonium chloride (EPTMAC)

\begin{tabular}{|c|c|c|c|c|c|c|c|c|c|c|}
\hline Sample & Method & Substrate & $\begin{array}{l}\mathrm{NaOH} \\
(\mathrm{M})\end{array}$ & Molar ratio & $\begin{array}{l}\text { Consistency } \\
(\%)\end{array}$ & $\begin{array}{l}\text { Temp. } \\
\left({ }^{\circ} \mathrm{C}\right)\end{array}$ & $\begin{array}{l}\text { Time } \\
\text { (h) }\end{array}$ & $\mathrm{DS}_{\mathrm{NMR}}$ & $\begin{array}{l}\mathrm{RE}^{\mathrm{a}} \\
(\%)\end{array}$ & $\mathrm{O}-2$ \\
\hline 1 & M1 & CL & 0.1 & 0.53 & 23 & 140 & 1 & 0.06 & 11.3 & 0.9 \\
\hline 2 & M1 & CL & 1 & 0.53 & 23 & 140 & 1 & 0.05 & 9.4 & 0.6 \\
\hline 3 & M1 & SKP & 0.1 & 0.53 & 23 & 140 & 1 & 0.06 & 11.3 & 0.8 \\
\hline 4 & M1 & SKP & 1 & 0.53 & 23 & 140 & 1 & 0.06 & 11.3 & 0.7 \\
\hline 5 & M2 & CL & 0.1 & 2.10 & 4.8 & 50 & 5 & 0.04 & 1.9 & 1.1 \\
\hline 6 & M2 & CL & 1 & 2.10 & 4.8 & 50 & 5 & 0.05 & 2.3 & 0.9 \\
\hline 7 & M2 & SKP & 0.1 & 2.10 & 4.8 & 50 & 5 & 0.06 & 2.8 & $1.0^{\mathrm{b}}$ \\
\hline 8 & M2 & SKP & 1 & 2.10 & 4.8 & 50 & 5 & 0.06 & 2.8 & 0.9 \\
\hline
\end{tabular}

The molar ratio is calculated as EPTMAC/AGU

${ }^{a}$ Reaction efficiency

b The relative amount of O-2 substitution, obtained by HSQC-NMR, of softwood kraft pulp (SKP) modified according to M2 is normalized to 1.0

\section{Influence of consistency and temperature using the spray-technique}

To improve on our knowledge of the spray-technique, further experiments varying the temperature and consistency were developed. In these experiments the amount of EPTMAC was increased to avoid the concentration step using preparative ion exchange chromatography, as there may be a possibility of cationic monomers not eluting from the column.

A study examining the difference between the methods using the same conditions were implemented, and the results are presented in Table 2.

As can be seen in Table 2, the highest degree of substitution and reaction efficiency is obtained using the spray-technique at $140{ }^{\circ} \mathrm{C}$ (sample 9). These results indicate that the procedure using an open bowl inside an oven at higher temperatures is beneficial for the etherification. It is however important to emphasize that the reaction temperature using method (M2) never exceeds $100{ }^{\circ} \mathrm{C}$ as the reaction occurs in a closed round bottomed flask containing an aqueous alkaline slurry. The low yield for this reaction (sample 10) is probably a result of hydrolysis of the epoxide, which should be favored by higher temperatures in alkaline environments. When using lower temperatures, on the other hand, the method M2 is the better choice. One explanation could be that the pre-activation step using the spray-technique is too weak to activate the hydroxy groups at lower temperatures. Higher temperatures are thus important for favorable outcomes using the spray-technique and epoxides.

Table 2 Comparison of methods on the etherification of softwood kraft pulp (SKP) with the cationic molecule 2,3-epoxypropyltrimethylammonium chloride (EPTMAC), using the spray-technique (M1) and a heterogeneous alkaline aqueous system (M2)

\begin{tabular}{lllllcrrr}
\hline Sample & Substrate & Method & $\begin{array}{l}\text { NaOH } \\
(\mathrm{M})\end{array}$ & $\begin{array}{l}\text { Consistency } \\
(\%)\end{array}$ & $\begin{array}{l}\text { Temp. } \\
\left({ }^{\circ} \mathrm{C}\right)\end{array}$ & $\begin{array}{l}\text { Time } \\
(\mathrm{h})\end{array}$ & $\begin{array}{c}\mathrm{DS}_{\mathrm{NMR}} \\
(\%)\end{array}$ \\
\hline 9 & SKP & M1 & 0.1 & 44 & 140 & 1 & $0.16^{\mathrm{b}}$ \\
10 & SKP & M2 & 0.1 & 44 & $140^{\mathrm{c}}$ & 1 & 0.05 & 2.3 \\
11 & SKP & M1 & 0.1 & 4.8 & 50 & 5 & $0.02^{\mathrm{b}}$ & 1.0 \\
7 & SKP & M2 & 0.1 & 4.8 & 50 & 5 & 0.06 & 2.8 \\
\hline
\end{tabular}

The molar ratio is 2.1 and calculated as EPTMAC/AGU

\footnotetext{
a Reaction efficiency

b Mean DS-value of three experiments

c The reaction temperature inside the reaction flask was measured to $100{ }^{\circ} \mathrm{C}$
} 
Table 3 Influence of consistency on the etherification of softwood kraft pulp (SKP) with the cationic molecule 2,3-epoxypropyltrimethylammoniumchloride (EPTMAC), using the spray-technique (M1)

\begin{tabular}{llllllrr}
\hline Sample & Substrate & $\begin{array}{l}\mathrm{NaOH} \\
(\mathrm{M})\end{array}$ & $\begin{array}{l}\text { Consistency } \\
(\%)\end{array}$ & $\begin{array}{l}\text { Temp. } \\
\left({ }^{\circ} \mathrm{C}\right)\end{array}$ & $\begin{array}{l}\text { Time } \\
(\mathrm{h})\end{array}$ & $\begin{array}{l}\mathrm{DS}_{\mathrm{NMR}} \\
(\%)\end{array}$ \\
\hline 9 & SKP & 0.1 & 44 & 140 & 1 & 0.16 \\
12 & SKP & 0.1 & 17 & 140 & 1 & 0.10 & 4.7 \\
13 & SKP & 0.1 & 4.8 & 140 & 1 & 0.08 \\
\hline
\end{tabular}

The molar ratio is 2.1 and calculated as EPTMAC/AGU

a Reaction efficiency

These results are in line with a previous study performed by Lund et al. (2011), comparing acetone and isopropanol in a similar crosslinking reaction using diepoxides, observing that increased temperatures and acetone resulted in higher crosslinking yields. The choice of temperature in the oven should preferably be high to promote faster diffusion, and reasonably also higher than the boiling point of the solvent to promote fast evaporation. A temperature above the melting point of EPTMAC is also believed to gain the rate of this reaction.

Triplets of experiments corresponding to samples number 9 and 11 were performed to investigate the reproducibility of the spray-technique. The arithmetic mean DS-value for sample number 9 was calculated to 0.16 (reported in Table 2) and the sample standard deviation 0.03 . For sample number 11 , the arithmetic mean DS-value was calculated to 0.020 (reported in Table 2) and the sample standard deviation 0.003 . These experiments indicate a good reproducibility of the spray-technique and are in line with the conclusions drawn.

Furthermore, different consistencies were studied to examine the impact of solvent on reaction efficiency for the spray-technique. A trend towards higher reaction efficiencies when less water is used can be seen in Table 3. The hydrolysis of the epoxide reagent, which is possible in the water/reagent mixture, seems to be less affecting when using the spray-technique at higher temperatures. The degree of substitution using method M1 at a consistency of $4.8 \%$ and $140{ }^{\circ} \mathrm{C}$ (sample 13) is higher compared to method M2 at the same consistency (sample 7). One possible explanation could be that, for the spray-technique M1, the reaction between the epoxide and water in the oven is impeded as the solvent concurrently will be removed by evaporation.
The intramolecular acetal formation possible during acidic hydrolysis of $O$-(2-hydroxalkyl)celluloses, are not evidently visible in the NMR spectra (Mischnick and Momcilovic 2010; Lee and Perlin 1984). One explanation could be that neutralization of the hydrolysate prior to removal of water disfavors acetal formation. Neither could substitutions on the formed hydroxyl groups of 2-hydroxypropyltrimethylammonium chloride (i.e. $\mathrm{O}-\mathrm{X}$ ) be clearly interpreted in the NMR spectra. The possibility of further hydroxyalkylations on $\mathrm{O}-\mathrm{X}$ could here be reduced because of the charged ammonium ion and electrostatic interactions. However, side products cannot be excluded since traces of peaks can be seen in the areas where predicted shifts are expected. A further investigation of these side products would require higher concentrations thereof followed by additional ${ }^{1} \mathrm{H}-{ }^{1} \mathrm{H}$ correlated spectroscopy (COSY) and Nuclear Overhauser effect spectroscopy (NOESY) NMR.

\section{Influence of macromolecular structure using the spray-technique}

In this study, we compared the solvent-minimizing spray-technique (M1) with the heterogeneous alkaline aqueous system (M2) on cellulosic materials with other macromolecular structures than SKP and cotton linters: mercerized cellulose, dissolving pulp and nanocrystalline cellulose. The aim of the study was to investigate reaction efficiencies using the spray-technique on materials with different pre-treatments and crystallinities.

Dissolving pulp and mercerization of cellulosic materials is known to enhance reactivity of the cellulosic material. In Tables 4 and 5, the results from cationization of these materials are presented showing that the reaction efficiency of the cationization performed on mercerized cellulose and dissolving 
Table 4 Etherification of mercerized pulp (MP) with the cationic molecule 2,3-epoxypropyltrimethylammonium chloride (EPTMAC), using the spray-technique (M1) and a heterogeneous alkaline aqueous system (M2)

\begin{tabular}{llllllrr}
\hline Sample & Method & Substrate & $\begin{array}{l}\mathrm{NaOH} \\
(\mathrm{M})\end{array}$ & $\begin{array}{l}\text { Consistency } \\
(\%)\end{array}$ & $\begin{array}{l}\text { Temp. } \\
\left({ }^{\circ} \mathrm{C}\right)\end{array}$ & $\begin{array}{l}\text { Time } \\
(\mathrm{h})\end{array}$ & $\begin{array}{c}\mathrm{DS}_{\mathrm{NMR}} \\
(\%)\end{array}$ \\
\hline 14 & M1 & MP & 0.1 & 44 & 140 & 1 & 11.9 \\
15 & M1 & MP & 0.1 & 4.8 & 140 & 1 & 0.25 \\
16 & M2 & MP & 0.1 & 4.8 & 50 & 5 & 0.11 \\
\hline
\end{tabular}

The molar ratio is 2.1 and calculated as EPTMAC/AGU

a Reaction efficiency

Table 5 Etherification of dissolving pulp (DP) with the cationic molecule 2,3-epoxypropyltrimethylammonium chloride (EPTMAC), using the spray-technique (M1) and the more common etherification method (M2)

\begin{tabular}{llllllrrr}
\hline Sample & Method & Substrate & $\begin{array}{l}\mathrm{NaOH} \\
(\mathrm{M})\end{array}$ & $\begin{array}{l}\text { Consistency } \\
(\%)\end{array}$ & $\begin{array}{l}\text { Temp. } \\
\left({ }^{\circ} \mathrm{C}\right)\end{array}$ & $\begin{array}{l}\mathrm{Time}^{2} \\
(\mathrm{~h})\end{array}$ & $\begin{array}{c}\mathrm{DS}_{\mathrm{NMR}} \\
(\%)\end{array}$ \\
\hline 17 & M1 & DP & 1 & 44 & 140 & 1 & 0.29 \\
18 & M1 & DP & 1 & 4.8 & 140 & 1 & 0.13 \\
19 & M2 & DP & 1 & 4.8 & 50 & 5 & 0.06 \\
\hline
\end{tabular}

The molar ratio is 2.1 and calculated as EPTMAC/AGU

a Reaction efficiency

pulp is higher than for SKP and cotton linters using the spray-technique. The results in Tables 4 and 5 demonstrate once again a high efficiency of the spraytechnique performed at $140{ }^{\circ} \mathrm{C}$ at high consistencies, showing the highest DS-value for both mercerized pulp and dissolving pulp. The DS-value for dissolving pulp using the heterogeneous alkaline aqueous system (M2) is however surprisingly low, and should be interpreted carefully.

Cationization of nanocrystalline cellulose, a highly crystalline cellulosic material, was also examined using the spray-technique. In the production of the nanocrystalline cellulose, the final step is freezedrying of the material. To keep the material in this firmly condition and hence be able to distribute the spraying onto the material in a fairly similar way as previous samples, the nanocrystalline cellulose was not activated in a sodium hydroxide solution. The results are presented in Table 6.

Doublets of experiments corresponding to samples number 20 and 21 were performed to investigate the reproducibility of the spray-technique on nanocrystalline cellulose. The mean DS-value for sample number 20 was calculated to $0.12 \pm 0.03$, and for sample $210.10 \pm 0.02$ (reported in Table 6).

The reactivity of the highly crystalline nanocrystalline cellulose using this method is somewhat lower compared to SKP, which probably is influenced by

Table 6 Etherification of nanocrystalline cellulose with the cationic molecule 2,3-epoxypropyltrimethylammonium chloride (EPTMAC), using the spray-technique (M1) and the more common etherification method (M2)

\begin{tabular}{llllllll}
\hline Sample & Substrate & $\begin{array}{l}\mathrm{NaOH} \\
(\mathrm{M})\end{array}$ & Molar ratio & $\begin{array}{l}\text { Consistency } \\
(\%)\end{array}$ & $\begin{array}{l}\text { Temp. } \\
\left({ }^{\circ} \mathrm{C}\right)\end{array}$ & $\begin{array}{l}\text { Time } \\
(\mathrm{h})\end{array}$ & $\begin{array}{c}\mathrm{DS}_{\mathrm{NMR}} \\
(\%)\end{array}$ \\
\hline 20 & NCC & - & 2.1 & 44 & 140 & 1 & 0.12 \\
21 & NCC & - & 2.1 & 4.8 & 140 & 1 & 0.10 \\
22 & NCC & 2 & 3 & 4.8 & 50 & 5 & $0.04^{\mathrm{b}}$ \\
\hline
\end{tabular}

The molar ratio is calculated as EPTMAC/AGU

a Reaction efficiency

b The experiment using M2 is reported in previous research (de la Motte et al. 2011) 
less activation; still the spray-technique could serve as an alternative cationization method for nanocrystalline cellulose.

\section{Investigation of regioselectivity using the spray-technique}

Previous research has shown that different etherification methods can influence the regioselectivity of the obtained products (Heinze and Pfeiffer 1999; Klemm 1998), and the observation that substitution at position $\mathrm{O}-2$ is comparatively high in heterogeneous reactions, because of its relatively minor involvement in the intermolecular hydrogen bonding pattern of the cellulose crystalline structure, has also been investigated previously (Volkert and Wagenknecht 2008).

During the evaluation of the two etherification methods, we observed a difference in regioselectivity. Comparison of the relative intensity of the integrals obtained from the HSQC spectra gave a relative change of the regioselectivity between the samples. The amount of O-2 substitution of SKP modified according to M2 was normalized to 1.0 (which, according to its HSQC spectrum, corresponds to $49 \%$ O-2 substitution). It is however important to emphasize that HSQC-NMR is appropriate for investigations of relative intensities and ratios of peaks, but not as a quantitative method. Although the percental O-2 substitution values obtained by HSQC are realistic, the slow relaxation of the indirectly detected

Table 7 Regioselectivities obtained by the spray-technique (M1) and the more common etherification method (M2)

\begin{tabular}{lllll}
\hline Substrate & Method & O-2 & Method & O-2 \\
\hline Softwood kraft pulp & M1 $^{\mathrm{a}}$ & $0.5^{\mathrm{b}}$ & $\mathrm{M}^{\mathrm{c}}$ & 1.0 \\
Mercerized SKP & $\mathrm{M} 1$ & 0.8 & $\mathrm{M} 2$ & 0.8 \\
Dissolving pulp & $\mathrm{M} 1$ & 0.9 & $\mathrm{M} 2$ & 0.9 \\
Nanocrystalline cellulose & $\mathrm{M} 1$ & $0.2^{\mathrm{b}}$ & $\mathrm{M}^{\mathrm{d}}$ & 0.8 \\
\hline
\end{tabular}

The relative amount of $\mathrm{O}-2$ substitution obtained by HSQCNMR, of softwood kraft pulp modified according to M2 is normalized to 1.0 , in accordance with Table 1

${ }^{a}$ Reaction conditions for M1: Consistency $44 \%, 140{ }^{\circ} \mathrm{C}, 1 \mathrm{~h}$, molar ratio 2.1

b Mean values

${ }^{\mathrm{c}}$ Reaction conditions for M2: Consistency $4.8 \%, 50{ }^{\circ} \mathrm{C}, 5 \mathrm{~h}$, molar ratio 2.1

d The experiment is reported in previous research (de la Motte et al. 2011) carbon nuclei decreases the reliability of the integrals slightly. The integral ratios, on the other hand, indicate the relative amount of various products formed or the ratio of products and starting material and can therefore be trusted.

The regioselectivity seemed to change from O-6 $\geq$ O- $2>0-3$ to O-6 $>$ O-2 $>$ O-3 when applying the spray-technique on SKP and cotton cellulose (Table 1). This observation was recognized in the following studies, showing a decrease in O-2 substitution when the spray-technique was applied on SKP. One explanation could be the reduced amount of alkali and water present during the spraying-method, which could result in more substitutions at position O-6, as the reaction will occur in a less swollen state.

However, the regioselectivity is maintained as O- $6 \geq 0$ O-2 $>$ O-3 for the alkali pretreated cellulosic samples using either of the methods (Table 7). Investigation of the crystalline regions of the mercerized pulp, dissolving pulp and SKP using X-ray diffraction (Fig. 3) proves the conversion from cellulose I to cellulose II during the mercerization, which could explain both the efficiency of and the regioselectivity. Mercerized pulp is more reactive than SKP and has a supramolecular order that allows more substitutions at position O-2.

On the contrary, the X-ray results display a similarity in crystallinity of SKP and dissolving pulp. The high reactivity and reduced regioselectivity of dissolving pulp is consequently considered to be a result of a lower degree of polymerization, faster diffusion and less hemicelluloses present.

Interestingly, the reactivity of position $\mathrm{O}-2$ in nanocrystalline cellulose is very low using this spray-technique compared to other cellulosic materials (Fig. 4) and previous experiments using a heterogeneous alkaline aqueous system (M2) (de la Motte et al. 2011). The sulphate groups located at the surface on nanocrystalline cellulose do not seem to aggravate the substitution at position O-6.

However, based on the experiences presented in this paper, a further investigation regarding the distribution of substituents within the fibers should be taken into consideration. Cellulose fibers are complicated in structure, and the reaction conditions applied using the spray-technique, i.e. less solvent and solvent evaporation during reaction, could result in a different distribution of substituents between the fiber bulk and surface. 


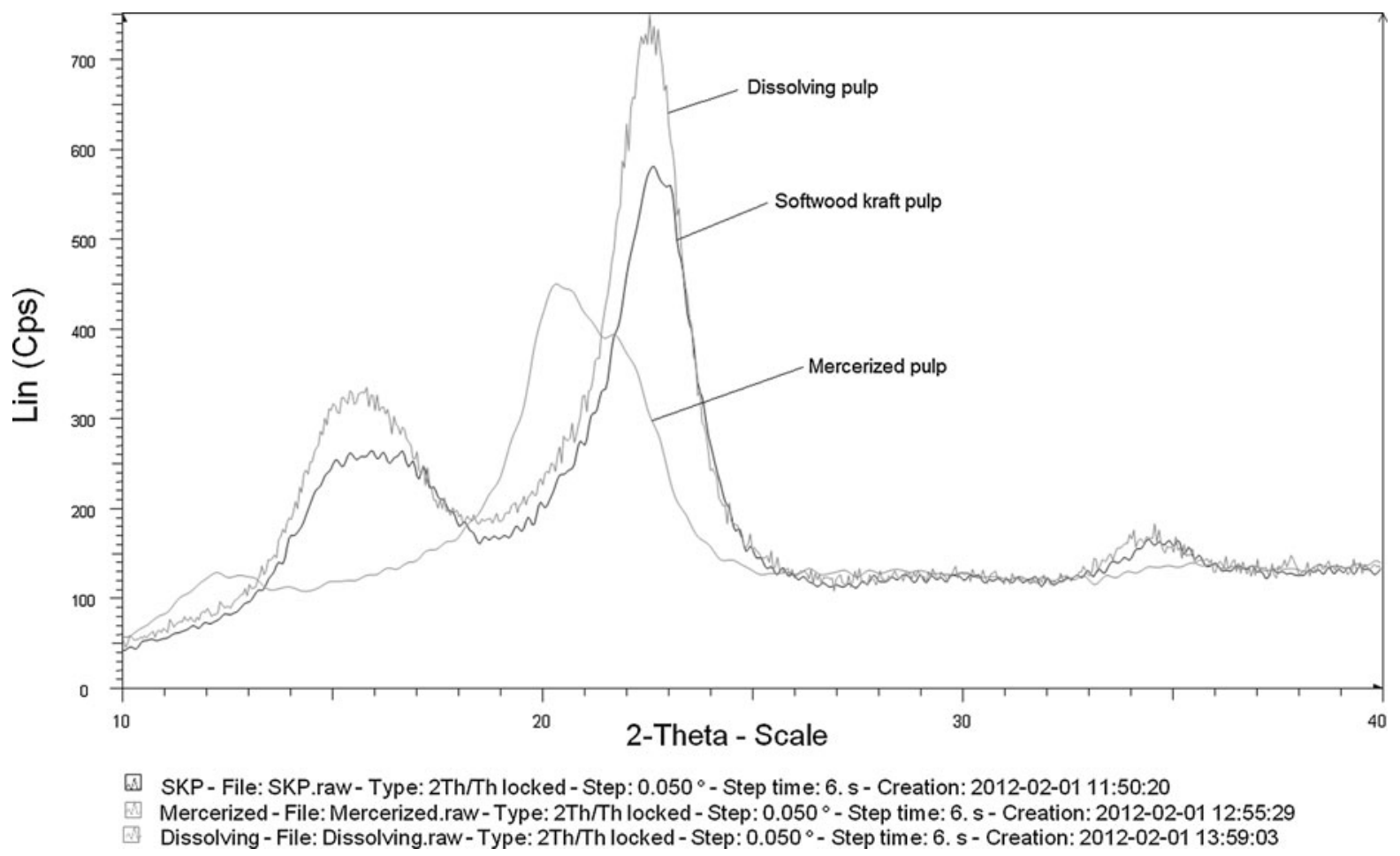

Fig. 3 X-ray diffraction results of SKP, mercerized pulp and dissolving pulp
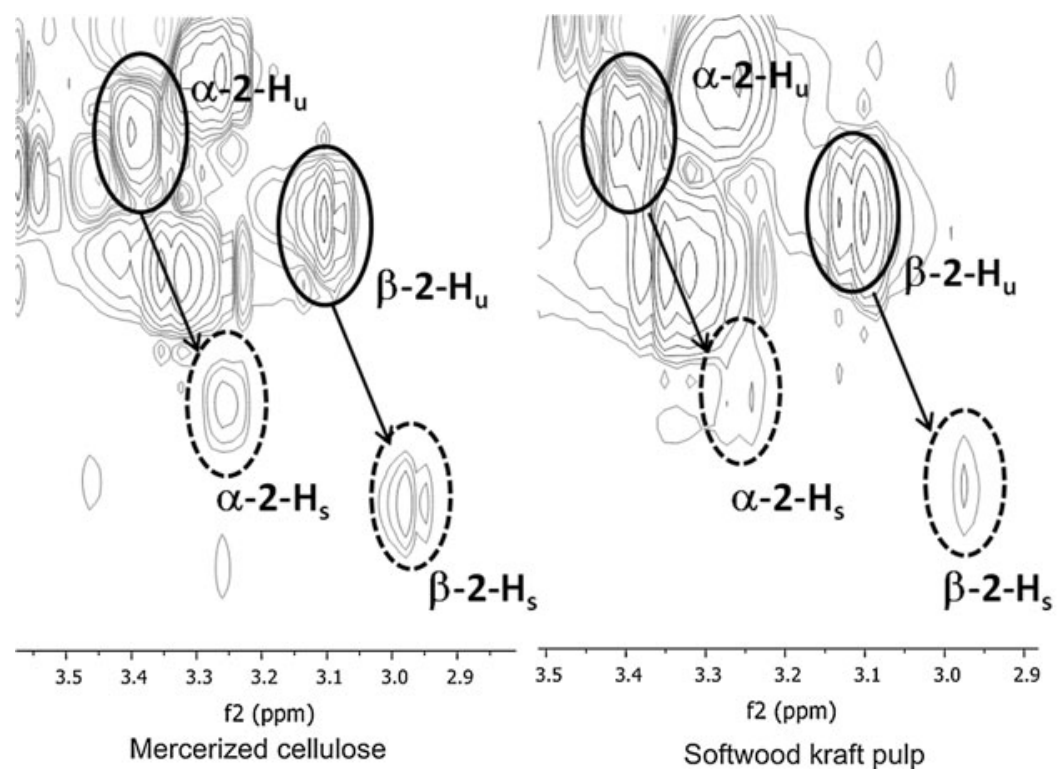

$3.5 \quad 3.4$

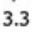

$\mathrm{f} 2$ (ppm)

Softwood kraft pulp

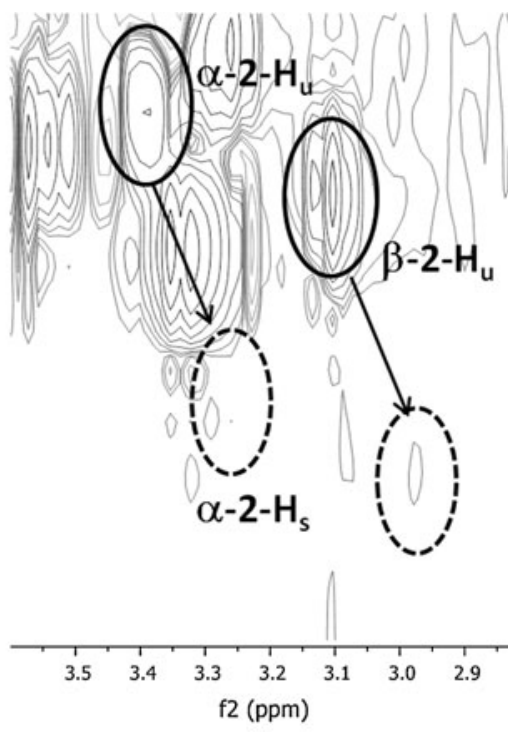

Nanocrystalline cellulose
Fig. 4 Comparison of HSQC-NMR spectra of mercerized cellulose, SKP, and nanocrystalline cellulose displaying the relative intensity of the protons located on the directly attached carbons to the un-substituted $\mathrm{O}-2\left(\mathrm{H}_{\mathrm{u}}-2\right)$ and substituted $\mathrm{O}-2$ $\left(\mathrm{H}_{\mathrm{s}}-2\right)$ positions. The samples are cationized using the spraytechnique (M1) at a consistency of $44 \%$, at $140{ }^{\circ} \mathrm{C}$ for $1 \mathrm{~h}$ 


\section{Conclusions}

Etherification of various cellulosic materials with the cationic epoxide 2,3-epoxypropyltrimethylammonium chloride (EPTMAC) was successfully accomplished using an efficient solvent-minimizing spraytechnique. The obtained materials was studied and evaluated regarding reaction efficiency and regioselectivity, using high resolution $1 \mathrm{D}$ and ${ }^{1} \mathrm{H}-{ }^{1} \mathrm{H}$ and ${ }^{1} \mathrm{H}-{ }^{13} \mathrm{C}$ correlated $2 \mathrm{D}$ NMR experiments. The results confirm a high efficiency of the spray-technique resulting in the highest degree of substitution $\left(\mathrm{DS}_{\mathrm{NMR}}\right)$ in this study. The spray-technique utilizes an open reaction bowl inside an oven to evaporate the solvent during the reaction, and an activation step with sodium hydroxide to avoid an alkaline aqueous environment in the spraying-mixture, which seem to be beneficial for this kind of etherifications. However, a lowering in the temperature or consistencies will have negative impacts on the rate of reaction for the spray-technique.

Furthermore, the spray-technique decreases substitutions at position $\mathrm{O}-2$, indicating a regioselectivity of O-6 $>$ O-2 $>$ O-3 for SKP and cotton linters. However, etherification using the spray-technique performed on mercerized cellulose, dissolving pulp and nanocrystalline cellulose, results in other efficiencies and regioselectivities. Mercerized cellulose and dissolving pulp are similarly more reactive towards the epoxide compared to SKP and cotton, and has a regioselectivity of $\mathrm{O}-6 \geq \mathrm{O}-2>\mathrm{O}-3$. Nanocrystalline cellulose, on the other hand, has the lowest reactivity and a regioselectivity of $0-6 \gg$ $\mathrm{O}-2>\mathrm{O}-3$.

The work presented on this etherification method expands the knowledge of the structure-property relationship in the synthesis of cationic cellulose ethers, verifying that different methods influence regioselectivity and material properties, and serves as a complement to recent research activities involving homogenous systems and control of regioselectivity.

Acknowledgments The authors would like to gratefully acknowledge VINNOVA, SCA Hygiene Products AB and Södra for financial support and for giving us the opportunity to work with this interesting project. Professsor Vratislav Langer, Department of Chemical and Biological Engineering, Environmental Inorganic Chemistry, Chalmers University of Technology, is acknowledged for performing the X-ray diffraction.
Open Access This article is distributed under the terms of the Creative Commons Attribution License which permits any use, distribution, and reproduction in any medium, provided the original author(s) and the source are credited.

\section{References}

Antal M, Micko M (1992) Preparation of microcrystalline cellulose aminoderivatives. Carbohydr Polym 19(3):167-169. doi:10.1016/0144-8617(92)90157-1

Baumann H, Liu C, Faust V (2003) Regioselectively modified cellulose and chitosan derivatives for mono- and multilayer surface coatings of hemocompatible biomaterials. Cellulose 10(1):65-74. doi:10.1023/a:1023084628101

Beck-Candanedo S, Roman M, Gray DG (2005) Effect of reaction conditions on the properties and behavior of wood cellulose nanocrystal suspensions. Biomacromolecules 6(2):1048-1054. doi:10.1021/bm049300p

Belalia R, Grelier S, Benaissa M, Coma V (2008) New bioactive biomaterials based on quaternized chitosan. J Agric Food Chem 56(5):1582-1588. doi:10.1021/jf071717+

Buschlediller G, Zeronian SH (1992) Enhancing the reactivity and strength of cotton fibers. J Appl Polym Sci 45(6): 967-979. doi:10.1002/app.1992.070450604

de la Motte H, Hasani M, Brelid H, Westman G (2011) Molecular characterization of hydrolyzed cationized nanocrystalline cellulose, cotton cellulose and softwood kraft pulp using high resolution 1D and 2D NMR. Carbohydr Polym 85(4):738-746. doi:10.1016/j.carbpol.2011.03.038

Fox SC, Li B, Xu D, Edgar KJ (2011) Regioselective esterification and etherification of cellulose: a review. Biomacromolecules 12(6):1956-1972. doi:10.1021/bm200260d

Hasani M, Westman G, Potthast A, Rosenau T (2009) Cationization of cellulose by using N-oxiranylmethyl-N-methylmorpholinium chloride and 2-oxiranylpyridine as etherification agents. J Appl Polym Sci 114(3):1449-1456. doi:10.1002/app.30548

Hashem M, Hauser P, Smith B (2003) Reaction efficiency for cellulose cationization using 3-chloro-2-hydroxypropyl trimethyl ammonium chloride. Text Res J 73(11): 1017-1023. doi:10.1177/004051750307301113

Heinze T, Liebert T (2001) Unconventional methods in cellulose functionalization. Prog Polym Sci 26(9):1689-1762. doi:10.1016/s0079-6700(01)00022-3

Heinze T, Petzold K (2008) Cellulose chemistry: novel products and synthesis paths. Elsevier, Amsterdam, pp 343-368. doi:10.1016/b978-0-08-045316-3.00016-8

Heinze T, Pfeiffer K (1999) Studies on the synthesis and characterization of carboxymethylcellulose. Angew Makromol Chem 266:37-45

Klemm D (1998) Regiocontrol in cellulose chemistry: principles and examples of etherification and esterification. ACS symposium series 688 (cellulose derivatives), p 19

Klemm D, Philipp B, Heinze T, Heinze U, Wagenknecht W (1998) Comprehensive cellulose chemistry, vol 1. WileyVCH, Weinheim 
Lee DS, Perlin AS (1984) Formation, and stereochemistry, of 1,2O-(1-methyl-1,2-ethanediyl)-D-glucose acetals formed in the acid-catalyzed hydrolysis of O-(2-hydroxypropyl)cellulose. Carbohydr Res 126(1):101-114. doi:10.1016/0008-6215(84) 85129-0

Lim SH, Hudson SM (2004) Synthesis and antimicrobial activity of a water-soluble chitosan derivative with a fiberreactive group. Carbohydr Res 339(2):313-319. doi: 10.1016/j.carres.2003.10.024

Lund K, de la Motte H, Brelid H, Westman G, Hanson C (2011) Diepoxide treatment of softwood kraft pulp: influence on absorption properties of fibre networks. Cellulose 18(5): 1365-1375. doi:10.1007/s10570-011-9560-9

Mischnick P, Momcilovic D (2010) Chemical structure analysis of starch and cellulose derivatives. In: Horton D (ed) Advances in carbohydrate chemistry and biochemistry, vol 64, pp 117-210. doi:10.1016/s0065-2318(10)64004-8

Peniche H, Peniche C (2011) Chitosan nanoparticles: a contribution to nanomedicine. Polym Int 60(6):883-889. doi: 10.1002/pi.3056
Petzold K, Koschella A, Klemm D, Heublein B (2003) Silylation of cellulose and starch - selectivity, structure analysis, and subsequent reactions. Cellulose 10(3):251-269. doi: 10.1023/a:1025173900225

Saito T, Isogai A (2007) Wet strength improvement of TEMPOoxidized cellulose sheets prepared with cationic polymers. Ind Eng Chem Res 46(3):773-780. doi:10.1021/ie0611608

Theander O, Westerlund EA (1986) Studies on dietary fiber. 3. Improved procedures for analysis of dietary fiber. J Agric Food Chem 34:330-336

Volkert B, Wagenknecht W (2008) Substitution patterns of cellulose ethers - Influence of the synthetic pathway. Macromol Symp 262:97-118

Yan LF, Tao HY, Bangal PR (2009) Synthesis and flocculation behavior of cationic cellulose prepared in a $\mathrm{NaOH} / \mathrm{urea}$ aqueous solution. Clean-Soil Air Water 37(1):39-44. doi: 10.1002/clen.200800127 